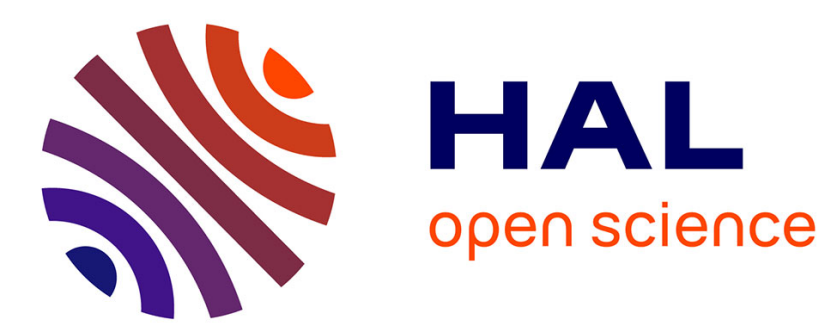

\title{
Double extensions of Lie algebras of Kac-Moody type and applications to some hamiltonian systems
}

Claude Roger

\section{To cite this version:}

Claude Roger. Double extensions of Lie algebras of Kac-Moody type and applications to some hamiltonian systems. 2012. hal-00705383

\section{HAL Id: hal-00705383 \\ https://hal.science/hal-00705383}

Preprint submitted on 7 Jun 2012

HAL is a multi-disciplinary open access archive for the deposit and dissemination of scientific research documents, whether they are published or not. The documents may come from teaching and research institutions in France or abroad, or from public or private research centers.
L'archive ouverte pluridisciplinaire HAL, est destinée au dépôt et à la diffusion de documents scientifiques de niveau recherche, publiés ou non, émanant des établissements d'enseignement et de recherche français ou étrangers, des laboratoires publics ou privés. 


\title{
Double extensions of Lie algebras of Kac-Moody type and applications to some hamiltonian systems
}

\author{
Claude Roger ${ }^{a}$
}

${ }^{a}$ Institut Camille Jordan ${ }^{1}$ Ecole Centrale de Lyon, INSA de Lyon, Université de Lyon, Université Lyon I, 43 boulevard du 11 novembre 1918, F-69622 Villeurbanne Cedex, France

Keywords: unimodular vector fields, extensions of Lie algebras, hydrodynamics,magnetohydrodynamics, coadjoint orbits of Lie algebras.

Mathematics Subject Classification (2000): 17B56, 22E66, 53D17, 58Z05,76W05.

\section{Abstract}

We shall describe some Lie algebras of Kac-Moody type, construct their double extensions, central and by derivations; in some cases, the corresponding Lie groups will also be constructed. The case of Lie algebra of unimodular vector fields will be studied in more details. We use the linear Poisson structure on their regular duals to construct generalizations of some infinite dimensional hamiltonian systems, such as magnetohydrodynamics.

\section{Introduction}

First of all, we define what we mean with Lie algebras of Kac-Moody type : they are Lie algebras $\mathfrak{g}_{\mathcal{A}}$ obtained by tensoring a Lie algebra $\mathfrak{g}$ over $\mathbb{K}$ ( a field of characteristic 0 ) with some associative and commutative $\mathbb{K}$-algebra $\mathcal{A}$, so $\mathfrak{g}_{\mathcal{A}}=\mathfrak{g} \otimes \mathcal{A}$, the bracket being naturally defined as : $[X \otimes a, Y \otimes b]=[X, Y] \otimes a b$. If $\mathbb{K}=\mathbb{C}, \mathfrak{g}$ is a simple Lie algebra and $\mathcal{A}=\mathbb{C}\left[t, t^{-1}\right]$ the algebra of Laurent polynomials, one obtains the loop algebra of $\mathfrak{g}$, usually denoted by $L(\mathfrak{g})$; for a more geometric version, we can complete the algebra and set $\mathcal{A}=C^{\infty}\left(S^{1}, \mathbb{C}\right.$ ), which allows identification (after suitable completion of the tensor product) $\mathfrak{g}_{\mathcal{A}}=C^{\infty}\left(S^{1}, \mathfrak{g}\right)$ the Lie algebra of $\mathfrak{g}$-valued currents on $S^{1}$.

The corresponding affine non-twisted Kac-Moody Lie algebra is then obtained through a double extension, with central term and outer derivation; let's construct first the universal central extension.

\footnotetext{
${ }^{1}$ Laboratoire associé au CNRS UMR 5208
} 


$$
1 \longrightarrow \mathbb{C} z \longrightarrow L \hat{(\mathfrak{g})} \longrightarrow L(\mathfrak{g}) \longrightarrow 1
$$

where $z$ stands for the central charge generating the 1-dimensional center, and then the extension by an outer derivation:

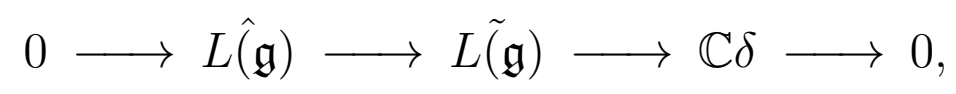

the generator $\delta$ of quotient term being the outer derivation $t \frac{d}{d t}$. A remarkable, and useful fact is that Killing form $\kappa$ for $\mathfrak{g}$ can naturally be extended to $L \tilde{(\mathfrak{g})}$ as $\mathcal{K}$ defined as follows:

$$
\mathcal{K}(f \otimes x, g \otimes y)=\kappa(x, y) \operatorname{Res}(f d g)
$$

or for the geometric version:

$$
\mathcal{K}(f \otimes x, g \otimes y)=\kappa(x, y) \int_{S^{1}} f d g
$$

one sets moreover:

$$
\mathcal{K}(z, \delta)=1
$$

all other terms vanishing. Those two extensions are compatible; they can be joined as a double extension $L \tilde{(\mathfrak{g})}$ as shown in the following diagram :

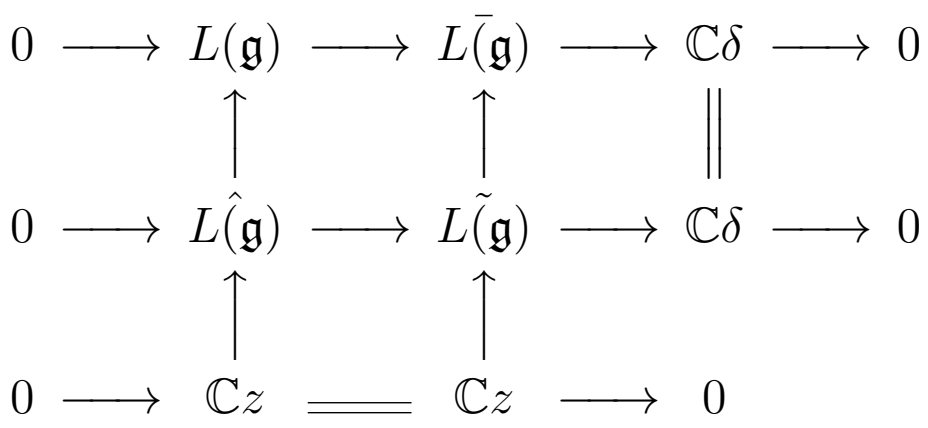

\section{Algebraic preliminaries}

Let's recall some elementary facts about cohomology of Lie algebras, cf.[13] for general theory and basic tools. One has for any Lie algebra $\mathfrak{g}$, a versal central extension:

$$
0 \longrightarrow H_{2}(\mathfrak{g}) \stackrel{i}{\longrightarrow} \hat{\mathfrak{g}} \stackrel{\pi}{\longrightarrow} \mathfrak{g} \longrightarrow 0 .
$$

Any cohomology class $c \in H^{2}(\mathfrak{g})=\operatorname{Hom}\left(H_{2}(\mathfrak{g}), \mathbb{K}\right)$ induces a central extension:

$$
0 \longrightarrow \mathbb{K} z \stackrel{i}{\longrightarrow} \tilde{\mathfrak{g}} \stackrel{\pi}{\longrightarrow} \mathfrak{g} \longrightarrow 0 .
$$

Let's remark that as a vector space $\tilde{\mathfrak{g}}=\mathfrak{g} \oplus \mathbb{K} z$ and its Lie bracket is defined as $[x+\lambda z, y+\mu z]=[x, y]+c(x, y) z$; the term versal simply means that any central 
extension can be obtained in this way. If moreover $H_{1}(\mathfrak{g})=0$, this extension(6) is universal, in the sense of universal (here, initial) object in the category of central extensions of $\mathfrak{g}$.

Let now $M$ be a $\mathfrak{g}$-module; the space $H^{1}(\mathfrak{g}, M)$ classifies derivations of $\mathfrak{g}$ with values in $M$ modulo inner ones. This result is particularly useful when $M=\mathfrak{g}$ with the adjoint representation; in such a case, a derivation is a map $\delta: \mathfrak{g} \rightarrow \mathfrak{g}$ such that

$$
\delta([X, Y])=[\delta(X), Y]+[X, \delta(Y)]
$$

while an inner derivation is given by the adjoint action of some element $Z \in \mathfrak{g}$. When $H^{1}(\mathfrak{g}, \mathfrak{g})$ is non trivial, one can construct the following extension by derivations

$$
0 \longrightarrow \mathfrak{g} \stackrel{i}{\longrightarrow} \overline{\mathfrak{g}} \stackrel{\pi}{\longrightarrow} H^{1}(\mathfrak{g}, \mathfrak{g}) \longrightarrow 0 .
$$

As a vector space, one has $\overline{\mathfrak{g}}=\mathfrak{g} \oplus H^{1}(\mathfrak{g}, \mathfrak{g})$, and the Lie bracket is the following: $\left[x+\delta_{1}, y+\delta_{2}\right]=[x, y]+\delta_{1}(y)-\delta_{2}(x)+\left[\delta_{1}, \delta_{2}\right]$, the last term being simply the commutator of derivations.

In order to generalize the construction of Kac-Moody type Lie algebras, one can try to construct double extensions as above[3]:

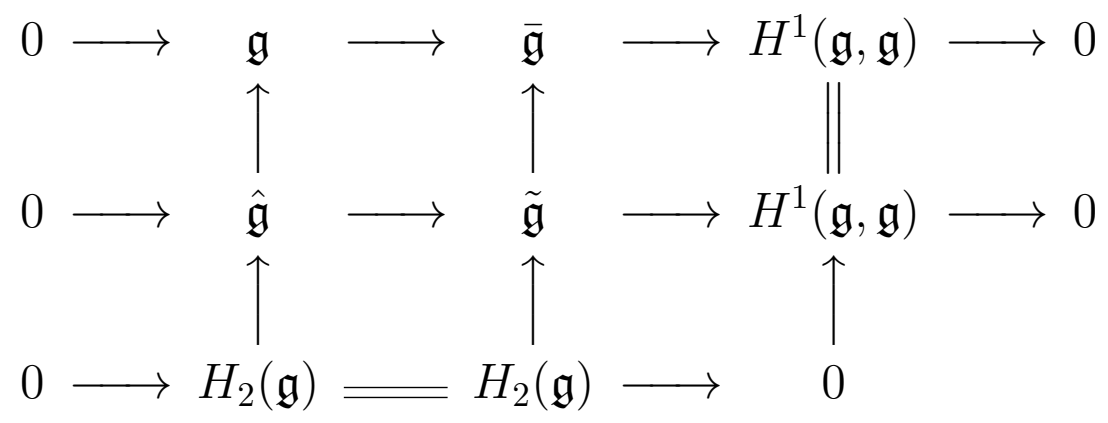

or, by singling out elements $c \in H^{2}(\mathfrak{g})$, and $\delta \in H^{1}(\mathfrak{g}, \mathfrak{g})$.

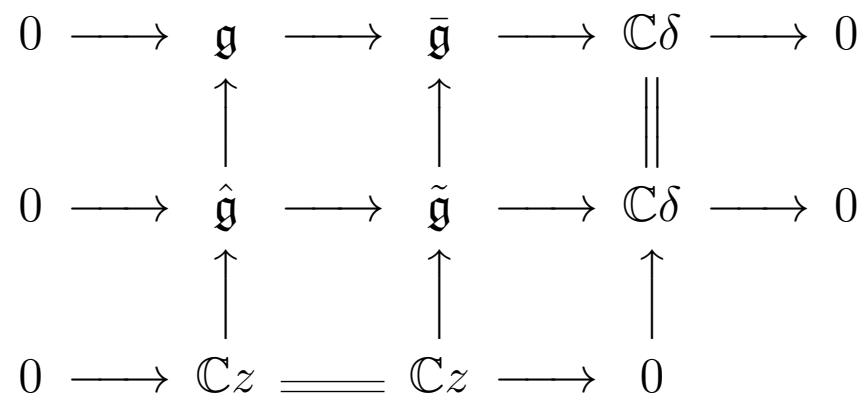

The double extension exists iff the following compatibility condition is satisfied:

$$
\text { For any } X, Y \in \mathfrak{g} \text {, one has } c(\delta X, Y)+c(X, \delta Y)=0 \text {. }
$$

Check it! 


\section{Main constructions for Lie algebras of Kac-Moody type}

We shall now work out the above constructions for the Lie algebras $\mathfrak{g}_{\mathcal{A}}$ defined in the introduction, using homological calculations by Haddi[10, 9] and Zusmanovich[31]. Their results imply the following:

$$
H_{2}\left(\mathfrak{g}_{\mathcal{A}}\right)=\left(H_{2}(\mathfrak{g}) \otimes \mathcal{A}\right) \oplus\left(B(\mathfrak{g}) \otimes H C_{1}(\mathcal{A})\right) \oplus(?),
$$

$H C_{1}(\mathcal{A})$ is the first cyclic homology group of $\mathcal{A}$ (cf. [20]), and $B(\mathfrak{g})=H_{0}\left(\mathfrak{g}, S^{2}(\mathfrak{g})\right)$ the space of symmetric coinvariants of degree 2 in $\mathfrak{g}$ (its dual, the space of symmetric bilinear invariants is much more popular), the term(?) vanishes when $\mathfrak{g}=[\mathfrak{g}, \mathfrak{g}](\mathfrak{g}$ is perfect). We shall explain later how to deduce explicit cocycles from those formulas.

If $\mathcal{A}$ is a smooth algebra, one has

$$
H C_{1}(\mathcal{A})=\Omega^{1}(\mathcal{A}) / d \mathcal{A}
$$

$\Omega^{1}(\mathcal{A})$ being the space of Kähler differentials of degree 1 on $\mathcal{A}[12]$. A typical example is $\mathcal{A}=C^{\infty}(\mathcal{X}, \mathbb{R})$ for an arbitrary compact orientable manifold $\mathcal{X}$, so one has $H C_{1}(\mathcal{A})=$ $\Omega^{1}(\mathcal{X}) / d \Omega^{0}(\mathcal{X})$. If $\mathfrak{g}$ is semi-simple, then $H_{2}(\mathfrak{g})=0$, and $B(\mathfrak{g})=H_{0}\left(\mathfrak{g}, S_{2}(\mathfrak{g})\right)$ is one dimensional, given by a contravariant version of Killing form (or Casimir element, if one prefers). For outer derivations, one can deduce the following result from [14] :

$$
H^{1}\left(\mathfrak{g}_{\mathcal{A}}, \mathfrak{g}_{\mathcal{A}}\right)=\left(\operatorname{Id}_{\mathfrak{g}} \otimes \operatorname{Der}(\mathcal{A})\right) \oplus\left(H^{1}(\mathfrak{g}, \mathfrak{g}) \otimes \mathcal{A}\right) .
$$

Here $I d_{\mathfrak{g}}$ denotes the space of scalar multiples of identity map of $\mathfrak{g}$ into itself.

If $\mathcal{A}=C^{\infty}(\mathcal{X}, \mathbb{R})$ then $\operatorname{Der}(\mathcal{A})=\operatorname{Vect}(\mathcal{X})$ the Lie algebra of smooth vector fields on $\mathcal{X}$, and $\mathfrak{g}$ is semi-simple one has simply:

$$
H^{1}\left(\mathfrak{g}_{\mathcal{A}}, \mathfrak{g}_{\mathcal{A}}\right)=\operatorname{Vect}(\mathcal{X})
$$

A vector field $\xi \in V e c t(\mathcal{X})$ acts as $L_{\xi}(f \otimes x)=L_{\xi}(f) \otimes x$. So for $\mathcal{A}=C^{\infty}(\mathcal{X}, \mathbb{R})$ and $\mathfrak{g}$ is semi-simple, one can construct extensions of both types

$$
\begin{gathered}
0 \longrightarrow \Omega^{1}(\mathcal{X}) / d \Omega^{0}(\mathcal{X}) \stackrel{i}{\longrightarrow} \hat{\mathfrak{g}}_{\mathcal{A}} \stackrel{\pi}{\longrightarrow} \mathfrak{g}_{\mathcal{A}} \longrightarrow 0 \\
0 \longrightarrow \mathfrak{g}_{\mathcal{A}} \stackrel{i}{\longrightarrow} \overline{\mathfrak{g}}_{\mathcal{A}} \stackrel{\pi}{\longrightarrow} \operatorname{Vect}(\mathcal{X}) \longrightarrow 0
\end{gathered}
$$

\section{Poisson structures on dual spaces}

The dual space $\mathfrak{g}^{*}$ of any Lie algebra $\mathfrak{g}$ admits a (linear) Poisson structure, whose symplectic leaves are the orbits of the coadjoint action of $G$; this allows applications of 
various aspects of Lie theory to hamiltonian equations, see[17] for a general exposition of orbits method, and $[1,16,15]$ for applications to dynamical systems. We shall explain here how Poisson structure on $\mathfrak{g}^{*}$ is modified by introduction of central terms or outer derivations as above; let $\hat{\mathfrak{g}}$ be the central extension associated to a cohomology class $c \in H^{2}(\mathfrak{g})$ and $\overline{\mathfrak{g}}$ the extension of $\mathfrak{g}$ by outer derivation $\delta$ such as compatibility condition above is satisfied; we denote the corresponding double extension by $\tilde{\mathfrak{g}}$.

Let's now fix the notations: the coadjoint action of $X \in \mathfrak{g}$ on $\alpha \in \mathfrak{g}^{*}$ gives $a d_{X}^{*}(\alpha)$. We shall then compute the coadjoint actions $\hat{a} d^{*}, \bar{a} d^{*}, \tilde{a} d^{*}$. One can associate to $c \in$ $H^{2}(\mathfrak{g})$, its Souriau cocycle $S_{c} \in H^{1}\left(\mathfrak{g}, \mathfrak{g}^{*}\right)$ : it is obtained naturally through Koszul's exact sequence

$$
0 \longrightarrow \mathfrak{g}^{*} \wedge \mathfrak{g}^{*} \stackrel{i}{\longrightarrow} \mathfrak{g}^{*} \otimes \mathfrak{g}^{*} \stackrel{\pi}{\longrightarrow} S^{2}\left(\mathfrak{g}^{*}\right) \longrightarrow 0 .
$$

One then easily computes:

$$
\hat{a} d_{X}^{*}(\alpha, Z)=\left(a d_{X}^{*}(\alpha)+Z S_{c}(X), 0\right)
$$

with $Z$ in the dual of central term, the latter acting trivially; so Souriau cocycle modifies coadjoint action by an affine term. Similarly, one can dualize $\delta$ to $\delta^{*} \in$ $\operatorname{Hom}\left(\mathfrak{g}^{*}, \mathfrak{g}^{*}\right)$, and one gets

$$
\bar{a} d_{(X, \lambda \delta)}^{*}(\alpha)=a d_{X}^{*}(\alpha)-\lambda \delta^{*}(\alpha)
$$

(we have restricted the action of $\bar{a} d^{*}$ to the invariant subspace $\mathfrak{g}^{*}$ ). Finally, one obtains for coadjoint action of the double extension:

$$
\tilde{a} d_{(X, \lambda \delta)}^{*}(\alpha, Z)=\left(a d_{X}^{*}(\alpha)+Z S_{c}(X)-\lambda \delta^{*}(\alpha), 0\right)
$$

\section{The case of unimodular vector fields}

Let $\mathcal{X}$ be an orientable manifold and $\omega$ a volume form on it; one then defines the Lie algebra of unimodular vector fields on $\mathcal{X}$ as follows:

$$
\overline{\operatorname{SVect}}(\mathcal{X})=\left\{X \in \operatorname{Vect}(\mathcal{X}) \mid L_{X}(\omega)=\operatorname{Div}(X) \omega=0\right\}
$$

One has the following exact sequence of Lie algebras :

$$
0 \longrightarrow \operatorname{SVect}(\mathcal{X}) \stackrel{i}{\longrightarrow} \overline{\operatorname{SVect}}(\mathcal{X}) \stackrel{\pi_{\omega}}{\longrightarrow} H_{d R}^{n-1}(\mathcal{X}) \longrightarrow 0
$$

One sets $\pi_{\omega}(X)=\left[i_{X} \omega\right]$, one checks easily that $\pi_{\omega}$ is a Lie algebra morphism into the abelian Lie algebra $H_{d R}^{n-1}(\mathcal{X})$. It follows from the results of [19] that $S V e c t(\mathcal{X})$ is 
the derived ideal of $\overline{S V e c t}(\mathcal{X})$ and is a perfect Lie algebra known as Lie algebra of exact unimodular vector fields; so, the abelian Lie algebra $H_{d R}^{n-1}(\mathcal{X})$ is the abelianized of $\overline{S V e c t}(\mathcal{X})$. Moreover, that exact sequence can be interpreted in terms of outer derivations, as follows :

$$
H^{1}(S V e c t(\mathcal{X}), S V e c t(\mathcal{X}))=H_{d R}^{n-1}(\mathcal{X})
$$

in other words, the above extension is an extension by derivations[19, 29, 11].

The Lie algebra $S V e c t(\mathcal{X})$ admits a universal central extension[25, 24, 23]:

$$
0 \longrightarrow H_{d R}^{n-2}(\mathcal{X}) \stackrel{i}{\longrightarrow} \operatorname{SVe\hat {ct}}(\mathcal{X}) \stackrel{\pi}{\longrightarrow} \operatorname{SVect}(\mathcal{X}) \longrightarrow 0
$$

the universal cocycle being :

$$
c(X, Y)=\left[i_{X} i_{Y} \omega\right],
$$

and so $H^{2}(S V e c t(\mathcal{X}))$ is isomorphic to $H_{d R}^{n-2}(\mathcal{X}, \mathbb{R})$, and to $H_{D R}^{2}(\mathcal{X})$ after Poincaré duality (cf. $[19,23]$ for proofs, cf. also [25] for some generalizations to various geometrical frameworks). One can further stick together the two extensions into a double one

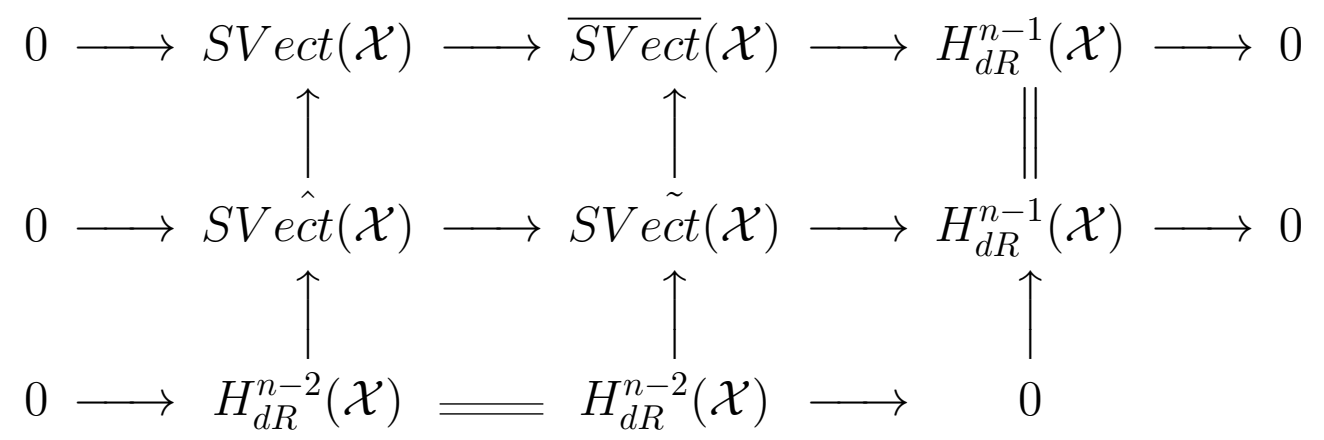

One need to prove the compatibility condition between cocycle and derivations: so let $\delta_{W}(X)=[X, W]$ the outer derivation of $S V e c t(\mathcal{X})$ given by bracket with some vector field $W \in \overline{S V e c t}(\mathcal{X})$; one computes easily:

$$
c\left(\delta_{W}(X), Y\right)=\left[i_{[X, W]} i_{Y} \omega\right]=\left[i_{X} L_{W} i_{Y} \omega-L_{W} i_{X} i_{Y} \omega\right]=\left[i_{X} L_{W} i_{Y} \omega\right]=\left[i_{[Y, W]} i_{X} \omega\right]=
$$

Hence the compatibility condition

$$
c\left(\delta_{W}(X), Y\right)+c\left(X, \delta_{W}(Y)\right)=0
$$

is satisfied.

We can remark now that those Lie algebras can be described by covariant tensors, using "musical " isomorphisms between contravariant and covariant tensor fields associated to volume form $\omega$ : for $\mathrm{X}$ and $\mathrm{Y}$ in $\overline{\operatorname{SVect}}(\mathcal{X})$, if $i_{X} \omega=\alpha, i_{Y} \omega=\beta$, we set $\alpha^{\sharp}=X, \beta^{\sharp}=Y$; we thus obtain identifications:

$$
Z^{n-1}(\mathcal{X}) \stackrel{\sharp}{\longrightarrow} \overline{S V e c t}(\mathcal{X})
$$




$$
\begin{gathered}
d \Omega^{n-2}(\mathcal{X}) \stackrel{\sharp}{\longrightarrow} \operatorname{SVect}(\mathcal{X}) \\
\Omega^{n-2}(\mathcal{X}) / d \Omega^{n-3}(\mathcal{X}) \stackrel{\sharp}{\longrightarrow} \operatorname{SVect}(\mathcal{X})
\end{gathered}
$$

For the case $n=2$ this is nothing but symplectic and hamiltonian vector fields on a surface, and $S V e c t(\mathcal{X})$ is easily identified to the corresponding Poisson algebra.

We shall mainly be interested here in the case $n=3$, which corresponds to the physical case of incompressible fluids in 3 dimensions. In that case, one can construct a kind of Killing form on $S V$ ect $(\mathcal{X})$, known as Arnold invariant: for $X, Y \in S V e c t(\mathcal{X})$, let $i_{X} \omega=d \alpha, i_{Y} \omega=d \beta$ with $\alpha, \beta \in \Omega^{1}(\mathcal{X})$. One then sets :

$$
\mathcal{J}(X, Y)=\int_{\mathcal{X}} \alpha \wedge d \beta
$$

It is easily checked that this bilinear mapping gives a well-defined, symmetric, non degenerate, and invariant form. Moreover, it can be extended into a Killing-like form to the doubly extended Lie algebra $S V e c t(\mathcal{X})$; simply use Poincaré duality to match $H_{D R}^{1}(\mathcal{X})$ and $H_{D R}^{2}(\mathcal{X})$ together. For dynamical interpretation and applications of Arnold invariant, see[1].

Before entering the applications, we shall describe briefly the dual spaces of those Lie algebras. But in this infinite dimensional situation one has to be careful: the purely algebraic duals being intractable, we shall restrict to linear maps given by smooth density measures. Here we have fixed the volume form $\omega$, so any 1-form $\eta$ defines a continuous linear map on $S V e^{-}{ }^{-}(\mathcal{X})$ as follows:

$$
<\eta, X>=\int_{\mathcal{X}} \eta(X) \omega
$$

If $\eta$ is exact, this form vanishes identically on $\overline{S V e c t}(\mathcal{X})$. So one has an identification:

$$
\overline{\operatorname{SVect}}(\mathcal{X})_{\text {reg }}^{*}=\Omega^{1}(\mathcal{X}) / d \Omega^{0}(\mathcal{X}) .
$$

If $\eta$ is closed and $X \in S V e c t(\mathcal{X})$, one has: $<\eta, X>=\int_{\mathcal{X}} \eta(X) \omega=-\int_{\mathcal{X}} \eta \wedge i_{X} \omega=$ $-\int_{\mathcal{X}} \eta \wedge d \alpha=\int_{\mathcal{X}} d(\eta \wedge \alpha)=0$. So one obtains an identification:

$$
S V \operatorname{Vect}(\mathcal{X})_{\text {reg }}^{*}=\Omega^{1}(\mathcal{X}) / Z^{1}(\mathcal{X})
$$

Analogous considerations would give :

$$
S \operatorname{Vect}(\mathcal{X})_{\text {reg }}^{*}=Z^{2}(\mathcal{X})
$$

For $n=3$, the covariant presentation of unimodular vector fields given above make isomorphisms between $S V e^{-} c t(\mathcal{X}), S V e c t(\mathcal{X})$ and their regular duals more natural, but 
the Arnold invariant $\mathcal{J}$, besides its geometrical flavor, gives an ad-invariant isomorphism

given by $\mathcal{J}^{\sharp}([u])=d u^{\sharp}$.

$$
\mathcal{J}^{\sharp}: S V e c t(\mathcal{X})_{\text {reg }}^{*} \rightarrow S V e c t(\mathcal{X}),
$$

\section{Higher order jets in Lie groups and magnetic extensions}

The notion of prolongation is well-known in differential geometry since the pionneering works of Elie Cartan in the 20's of last century, the idea being to extend the bundle of frames on a manifold to bundles of higher order jets of frames. One can associate to any Lie group $G$ the group of its $k$-th order frames $T^{(k)} G$, with forgetful maps $T^{(k)} G \rightarrow T^{\left(k^{\prime}\right)} G$ if $k>k^{\prime}$, and passing to projective limits, the bundle of frames of infinite order $T^{(\infty)} G$.

For their Lie algebras, the situation can be described in a purely algebraic way; let $T^{(k)} \mathfrak{g}=\operatorname{Lie}\left(T^{(k)} G\right)$, then $T^{(k)} \mathfrak{g}=\mathfrak{g}_{\mathcal{A}_{k}}$ as above, where $\mathcal{A}_{k}=\mathbb{K}[t] /\left(t^{k+1}\right)$. The two extreme cases are particularly meaningful ; one one hand $T^{(\infty)} \mathfrak{g}=\mathfrak{g} \otimes \mathbb{K}[[t]]$ is the Lie algebra of infinite jets, and $\operatorname{Lie}(T G)=T \mathfrak{g}=T^{(1)} \mathfrak{g}=\mathfrak{g}_{\mathcal{A}_{1}}$, the Lie algebra of the tangent group of $G$ on the other; usually $\mathcal{A}_{1}=\mathbb{K}[t] /\left(t^{2}\right)$ is called the ring of dual numbers on $\mathbb{K}$. One has moreover an isomorphism $T \mathfrak{g}=\mathfrak{g} \ltimes \mathfrak{g}_{a b}$, the semidirect product of $\mathfrak{g}$ with itself as $\mathfrak{g}$-module considered as an abelian Lie algebra.

If $\mathfrak{g}$ admits a Killing form, then $\mathfrak{g}=\mathfrak{g}^{*}$ as a $\mathfrak{g}$-module, and tangent and cotangent bundles on $G$ are isomorphic; so $T \mathfrak{g}$ and $T^{*} \mathfrak{g}$ are isomorphic as Lie algebras . It is called magnetic extension of $\mathfrak{g}$ (the term was initiated by Marsden cf.[21]) for reasons which will become apparent in the next section. Finally the Killing form $\kappa$ can be extended to $T \mathfrak{g}$, as $\kappa(X+t x, Y+t y)=\kappa(X, y)+\kappa(x, Y)$; the same trick works as well for $T^{(k)} \mathfrak{g}$, setting $\kappa\left(\Sigma_{0}^{k} X^{i} t^{i}, \Sigma_{0}^{k} Y^{j} t^{j}\right)=\Sigma_{i+j=k} \kappa\left(X^{i}, Y^{j}\right)$

We can now work out the constructions of section 3 above(assuming $\mathfrak{g}$ is perfect); one has $H C^{1} \mathcal{A}_{k}=0$, for $k \neq \infty$ (cf. appendix below). So:

$$
H_{2}\left(T^{(k)} \mathfrak{g}\right)=H_{2}(\mathfrak{g}) \otimes \mathcal{A}_{k}
$$

and

$$
H^{2}\left(T^{(k)} \mathfrak{g}\right)=H^{2}(\mathfrak{g}) \otimes \mathcal{A}_{k}^{\prime}
$$

It is now easy to see that $\operatorname{Der}\left(\mathcal{A}_{k}\right)=I(d / d t)$, where $I=(t)$ the maximal ideal of the local ring $\mathcal{A}_{k}, \operatorname{Der}\left(\mathcal{A}_{k}\right)$ is a nilpotent Lie algebra; in particular $\operatorname{Der}\left(\mathcal{A}_{1}\right)$ is one dimensional, generated by $t d / d t$. So one has:

$$
H^{1}\left(T^{(k)} \mathfrak{g}, T^{(k)} \mathfrak{g}\right)=\left(I d_{\mathfrak{g}} \otimes \operatorname{Der}\left(\mathcal{A}_{k}\right)\right) \oplus\left(H^{1}(\mathfrak{g}, \mathfrak{g}) \otimes \mathcal{A}_{k}\right)
$$


For the case $k=\infty, \operatorname{Der}\left(\mathcal{A}_{\infty}\right)=\mathbb{K}[[t]] d / d t$, the Lie algebra of formal vector fields in one indeterminate; and $H C^{1} \mathcal{A}_{\infty}=\Omega^{1}(\mathbb{K}[[t]]) / d \mathbb{K}[[t]]=0$; so one gets

$$
H_{2}\left(T^{(\infty)} \mathfrak{g}\right)=H_{2}(\mathfrak{g}) \otimes \mathbb{K}[[t]] .
$$

and

$$
H^{1}\left(T^{(\infty)} \mathfrak{g}, T^{(\infty)} \mathfrak{g}\right)=\left(I d_{\mathfrak{g}} \otimes \mathbb{K}[[t]] d / d t\right) \oplus\left(H^{1}(\mathfrak{g}, \mathfrak{g}) \otimes \mathcal{A}_{\infty}\right) .
$$

Let's study now some particular cases, and compute explicitly the corresponding modification of Poisson structures on the duals, associated to central or derivation terms as we computed in section 4 above. Remark first that $\mathcal{A}_{k}^{\prime}$ is isomorphic to $\mathcal{A}_{k}$ as $\mathbb{K}$-vector space; we shall denote by $\tau$ the dual variable to $t$.

If $G$ is semi-simple, then $H^{1}\left(T^{(1)} \mathfrak{g}, T^{(1)} \mathfrak{g}\right)=I d_{\mathfrak{g}} \otimes \mathbb{R}(\delta)$; explicitly, the latter derivation acts as $\delta(X+t x)=t x$, and on the dual level $\delta^{*}(A+\tau a)=\tau a$. The coadjoint action is then modified the following way:

$$
\bar{a} d_{(X+t x, \lambda \delta)}^{*}(A+\tau a)=a d_{X}^{*}(A)+a d_{x}^{*}(a)+\tau\left(a d_{X}^{*}(a)-\lambda a\right) ;
$$

the group $G$ being semi-simple, one has $H_{2}\left(T^{(1)} \mathfrak{g}\right)=0$, so the formula above gives the only available modification; we have obtained a kind of "toy model".

We shall now consider the case of $\mathfrak{g}=S V e c t(\mathcal{X})$ : one deduce from computations above

$$
H^{2}\left(T^{1} S V e c t(\mathcal{X})\right)=H^{2}(S V e c t(\mathcal{X})) \otimes \mathcal{A}_{1}^{\prime}=H_{d R}^{2}(\mathcal{X}) \otimes \mathcal{A}_{1}^{\prime}
$$

It is straightforward to deduce from the description of the universal central extension of $S V e c t(\mathcal{X})$ defined above, the central cocycle associated to a cohomology class $[H] \in$ $H_{d R}^{2}(\mathcal{X})$ of $H \in \Omega^{2}(\mathcal{X})$. One has:

$$
c_{[H]}(X, Y)=\int_{\mathcal{X}} H \wedge i_{X} i_{Y} \omega=\int_{\mathcal{X}} H(X, Y) \omega
$$

An equivalent formula is the following one: consider a codimension 2 submanifold $N \in \mathcal{X}$, such that its homology class $[N] \in H_{n-2}(\mathcal{X})$ is the Poincaré dual of $[H]$; then(cf.[29], Prop.2):

$$
c_{[N]}(X, Y)=\int_{[N]} \omega(X, Y) .
$$

And now for $T^{1} S V e c t(\mathcal{X})$, let $[H]+\tau[h] \in H_{d R}^{2}(\mathcal{X}) \otimes \mathcal{A}_{1}^{\prime}$, then

$$
c_{[H]+\tau[h]}(X+\tau x, Y+\tau y)=\int_{\mathcal{X}}(H(X, Y)+h(x, Y)+h(X, y)) \omega .
$$

One can now easily deduce from those formulas the expression of corresponding Souriau cocycle; recall that we have determined the regular dual

$$
S \operatorname{Vect}(\mathcal{X})_{\text {reg }}^{*}=\Omega^{1}(\mathcal{X}) / Z^{1}(\mathcal{X})
$$




$$
\text { so: } T^{1} S V e c t(\mathcal{X})_{\text {reg }}^{*}=\left(\Omega^{1}(\mathcal{X}) / Z^{1}(\mathcal{X})\right) \otimes \mathcal{A}_{1}^{\prime}
$$

We shall obtain

$$
S_{[H]+\tau[h]} \in H^{1}\left(T^{1} S V e c t(\mathcal{X}),\left(\Omega^{1}(\mathcal{X}) / Z^{1}(\mathcal{X})\right) \otimes \mathcal{A}_{1}^{\prime}\right),
$$

defined by

$S_{[H]+\tau[h]}(X+t x)=\left[i_{X} H+i_{x} h\right]+\tau\left[i_{X} h\right]$

(Warning: on the right hand side, the brackets indicate equivalence classes of 1forms modulo closed ones). One can now write down explicitly the coadjoint action in the extended Lie algebra as in section $\mathbf{4}$, formula (4.2):

$$
\hat{a} d_{X+t x}^{*}(A+\tau a, Z+\tau z)=a d_{X}^{*}(A)+a d_{x}^{*}(a)+Z\left[i_{X} H+i_{x} h\right]+\tau\left(a d_{X}^{*}(a)+z\left[i_{X} h\right]\right),
$$

where $Z+\tau z$ corresponds to central charge associated to cocycle $[H]+\tau[h]$.

Let's now give the terms corresponding to outer derivations: for the case of $\mathfrak{g}=$ $S V \operatorname{ect}(\mathcal{X})$, the computations above yield:

$H^{1}\left(T^{(1)} S V e c t(\mathcal{X}), T^{(1)} S V e c t(\mathcal{X})\right)=\left(I_{\text {SVect }(\mathcal{X})} \otimes \mathbb{R}(\delta)\right) \oplus\left(H^{1}(\operatorname{SVect}(\mathcal{X}), S V e c t(\mathcal{X})) \otimes \mathcal{A}_{1}\right)$.

and

$$
H^{1}(S V e c t(\mathcal{X}), S V e c t(\mathcal{X}))=H_{d R}^{n-1}(\mathcal{X})
$$

So finally:

$$
H^{1}\left(T^{(1)} \operatorname{SVect}(\mathcal{X}), T^{(1)} \operatorname{SVect}(\mathcal{X})\right)=\left(I d_{S V e c t}(\mathcal{X}) \otimes \mathbb{R}(\delta)\right) \oplus\left(H_{d R}^{n-1}(\mathcal{X}) \otimes \mathcal{A}_{1}\right),
$$

and

$$
H^{1}\left(T^{(1)} \overline{\operatorname{SVect}(\mathcal{X})}, T^{(1)} \overline{\operatorname{SVect}(\mathcal{X}))}=\left(I_{\text {SVect }(\mathcal{X})} \otimes \mathbb{R}(\delta)\right) .\right.
$$

We can now write the coadjoint action modified by those outer derivations(cf. formula $(4.3))$ :

$\bar{a} d_{(X+t x, \lambda \delta+U+t W)}^{*}(A+\tau a)=a d_{X}^{*}(A)+a d_{x}^{*}(a)-U^{*}(A)+\tau\left(a d_{X}^{*}(a)-U^{*}(a)-W^{*}(A)-\lambda a\right)$.

Here, $U+t W \in H_{d R}^{n-1}(\mathcal{X}) \otimes \mathcal{A}_{1}$; let's recall that in this context, the cohomology group $H_{d R}^{n-1}(\mathcal{X})$ appears as a quotient of $\overline{S V e c t}(\mathcal{X})$ by $S V e c t(\mathcal{X})$, so $U$ and $W$ can be considered as classes of vector fields in $\overline{\operatorname{SVect}}(\mathcal{X})$ modulo $S V$ ect $(\mathcal{X})$. 
In section 5 above, we have obtained a geometric characterization of regular duals, so $A$ and $a$ are identified with classes of 1 forms $[u]$ and $[b]$ in $\Omega^{1}(\mathcal{X}) / Z^{1}(\mathcal{X})$ and the coadjoint action above can be made more explicit, being identified with Lie derivative on differential forms. One gets :

$$
\bar{a} d_{(X+t x, \lambda \delta+U+t W)}^{*}([u]+\tau[b])=\left[L_{X} u+L_{x} b-L_{U} u\right]+\tau\left[L_{X} b-L_{U} b-L_{W} u-\lambda b\right],
$$

and for the centrally extended case considered above,

$$
\hat{a} d_{X+t x}^{*}([u]+\tau[b], Z+\tau z)=\left[L_{X} u+L_{x} b\right]+Z\left[i_{X} H+i_{x} h\right]+\tau\left(\left[L_{X} b\right]+z\left[i_{X} h\right]\right) .
$$

\section{The groups of diffeomorphisms associated to our extensions}

It is not always possible to integrate an infinite dimensional Lie algebra to a group, even in the Banach case. In our case the group of volume preserving diffeomorphisms $\overline{S \operatorname{Diff}(\mathcal{X})}$ is a Fréchet Lie group, which integrates Lie algebra $\overline{S V e c t}(\mathcal{X})$ (in a weak sense), and the Lie algebra mapping

$$
\overline{\operatorname{SVect}}(\mathcal{X}) \stackrel{\pi_{\omega}}{\longrightarrow} H_{d R}^{n-1}(\mathcal{X}) \longrightarrow 0
$$

can be integrated to a group homomorphism

$$
S \operatorname{Diff}(\mathcal{X}) \stackrel{\tilde{\Pi}_{\omega}}{\longrightarrow} H_{d R}^{n-1}(\mathcal{X}),
$$

where $S \operatorname{Dif} f(\mathcal{X})$ is the universal covering of the connected component of the identity $\overline{\operatorname{SDiff}}(\mathcal{X})_{0} \subset \overline{\operatorname{SDiff}}(\mathcal{X})$.

The group homomorphism $\tilde{\Pi}_{\omega}$, called flux, was first defined by W. Thurston (unpublished), see $[2,29]$ for details . Set $\Lambda_{\omega}=\tilde{\Pi}_{\omega}\left(\pi_{1}(\overline{\operatorname{SDiff}(\mathcal{X})})\right)$ be the image of

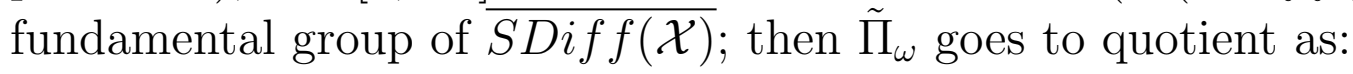

$$
\overline{\operatorname{SDiff}}(\mathcal{X})_{0} \stackrel{\Pi_{\omega}}{\longrightarrow} H_{d R}^{n-1}(\mathcal{X}) / \Lambda_{\omega} .
$$

One can show that $\Lambda_{\omega}$ is discrete, so the quotient group is Hausdorff, if volume form $\omega$ has integer periods(see again $[2,29]$ for details and various geometric interpretations). Now we have to deal with the problem of integration of central extensions, and we shall use once more the results of [29]. The flux homorphism can be generalized to differential forms of any degrees, and for 2-cocycles is the same as Souriau cocycle defined in the previous part : let $c$ be a 2-cocycle on $\overline{S V e c t}(\mathcal{X})$, the infinitesimal flux 
associated to it will be $s_{c}: \overline{\operatorname{SVect}}(\mathcal{X}) \rightarrow \overline{\operatorname{SVect}}(\mathcal{X})_{\text {reg }}^{*}$ defined as $s_{c}(X)=i_{X} c$. It can be integrated directly on the universal covering group as:

$$
\mathcal{S}_{c}: S \operatorname{Diff}(\mathcal{X}) \rightarrow \overline{\operatorname{SVect}}(\mathcal{X})_{\text {reg }}^{*}
$$

given by

$$
\mathcal{S}_{c}([\gamma])(X)=-\int_{\gamma} i_{X^{*}} c^{*},
$$

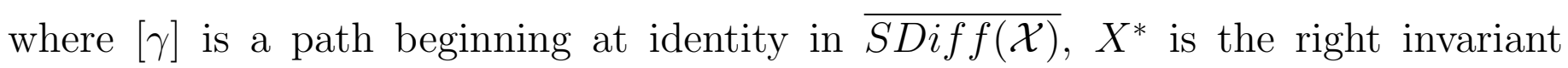
vector field on the group associated to $X$, and $c^{*}$ the left invariant form on the group associated to $c$ (cf.[29], part 3 for details).

Let's now restrict this flux homomorphism to the fundamental group, one gets: $\mathcal{S}_{c}: \pi_{1}(\operatorname{SDiff}(\mathcal{X})) \rightarrow H_{1}(\overline{S V e c t}(\mathcal{X}))=H_{n-1}(\mathcal{X})$. If one has $c=c_{[N]}$ associated to a $n$-2-dimensional manifold $N \subset \mathcal{X}$ as above, the flux satisfies:

$$
\mathcal{S}_{c_{[N]}}: \pi_{1}(S \operatorname{Diff}(\mathcal{X})) \rightarrow H_{1}(\overline{\operatorname{SVect}}(\mathcal{X}))=H_{n-1}(\mathcal{X})
$$

given by:

$$
\mathcal{S}_{c_{[N]}}\left(\left[\gamma_{t}\right]\right)=[\gamma N] \in H_{n-1}(\mathcal{X})
$$

where $[\gamma N]$ is an $n-1$-cycle in $\mathcal{X}$, defined as $[\gamma N](t, x)=\gamma_{t}(x) \in \mathcal{X}$ for $(t, x) \in$ $[0,1] \times N($ cf. again[29]).

Another obstruction to integrability from Lie algebra to Lie groups depends on homotopy group of degree 2 of the group of diffeomorphisms. Let $G$ be one of the groups of volume preserving diffeomorphisms considered above, one associates to a 2-cocycle $c$ its period homomorphism

$$
\operatorname{per}_{c}: \pi_{2}(G) \rightarrow \mathbb{R}
$$

defined by

$$
\operatorname{per}_{c}([\sigma])=\int_{S^{2}} \sigma^{*}\left(c^{*}\right)
$$

where $\sigma: S^{2} \rightarrow G$ represents the homotopy class, and $c^{*}$ is the left invariant 2 -form on $G$ associated to $c$ as above. The period group of $c$ is then $\Gamma_{c}=\operatorname{Im}\left(\right.$ per $\left._{c}\right)$. The final result is the following:

Theorem(Neeb cf.[29] Thm. 5):

If $\Gamma_{c}$ is discrete, and the flux homomorphism $S_{c}$ vanishes, then one has central extension of Lie groups:

$$
1 \longrightarrow \mathbb{R} / \Gamma_{c} \longrightarrow \hat{G} \longrightarrow G \longrightarrow 1
$$


integrating the extension of Lie algebras corresponding to cocycle $c$.

When $c=[H]$ associated to a 2-form $H$, then one sees easily that $\Gamma_{[H]}$ is contained in the group of periods of $H$. So if the periods of $H$ are integral, $\Gamma_{[H]}$ is discrete, this is the case in particular when $c=c_{[N]}$ for a submanifold $N \subset \mathcal{X}$.

As outcome we can conclude that integration of various extensions of Lie algebras considered in part 5 has some price, of topological nature: the groups $\Gamma_{c}$ and $\Lambda_{\omega}$ must be discrete, and the flux $S_{c}$ must vanish. Computing homotopy groups $\pi_{1}(G)$ and $\pi_{2}(G)$ for our diffeomorphisms groups $G$ is certainly a hard topological problem, on which very few is known, as far as we know. We are able to decide of triviality of $S_{c}$ in obvious cases: since $S V$ ect $(\mathcal{X})$ ) is perfect[19], its 1-homology vanishes, so the flux homomorphism vanishes for $\operatorname{SDiff}(\mathcal{X})$, as well as for universal covering $\operatorname{SDiff} f(\mathcal{X})$, its fundamental group being trivial by definition.

$$
\begin{aligned}
& 1 \longrightarrow \mathbb{R} / \Gamma_{c_{[N]}} \longrightarrow \operatorname{SDifff}(\mathcal{X}) \longrightarrow \operatorname{SDiff}(\mathcal{X}) \longrightarrow 1, \\
& 1 \longrightarrow \mathbb{R} / \Gamma_{c_{[N]}} \longrightarrow \operatorname{SDi} \hat{\tilde{f}} f(\mathcal{X}) \longrightarrow \operatorname{SDiff} f(\mathcal{X}) \longrightarrow 1 \text {. }
\end{aligned}
$$

Those central extensions corresponds to specializations of vertical arrows of diagram (5.4), since the universal covering $S \operatorname{Dif} f(\mathcal{X})$ integrates Lie algebra $\overline{S V e c t}(\mathcal{X})$. In fact extension (7.6) can be refined as follows: let $K_{c}$ be the kernel of the flux homomorphism, then one can centrally extend the quotient $S D i \hat{\tilde{f}} f(\mathcal{X}) / K_{c}$, which ist the best possible refinement. If moreover the volume form $\omega$ is integral, one has a group extension which integrates (5.1):

$$
1 \longrightarrow \operatorname{SDiff}(\mathcal{X}) \longrightarrow \overline{\operatorname{Siff}}(\mathcal{X})_{0} \stackrel{\Pi_{\omega}}{\longrightarrow} H_{d R}^{n-1}(\mathcal{X}) / \Lambda_{\omega} \longrightarrow 1 .
$$

This sequence is an abelianization exact sequence, so commutators

$$
\left[\overline{S \operatorname{Diff}}(\mathcal{X})_{0}, \overline{\operatorname{SDiff}}(\mathcal{X})_{0}\right] \subset \operatorname{SDiff}(\mathcal{X})
$$

in fact those two subgroups are equal up perhaps to some discrete quotient.

We must mention here, although we shall not need it, the very smart argument used in[29] part 4 to construct central extensions for those groups, using geometrical description of singular dual $\overline{S V e c t}(\mathcal{X})_{\text {sing }}^{*}$ through isotopy classes of 2-codimensional submanifolds, or generalized knots, and their geometrical quantization.

The last step of our construction of groups doesn't present major difficulties, since the tangent or cotangent Lie algebras can be easily integrated, $T^{(1)} \operatorname{SVect}(\mathcal{X})$ being the Lie algebra of $T^{(1)} \operatorname{SDiff}(\mathcal{X})=\operatorname{SDiff}(\mathcal{X}) \ltimes S V e c t(\mathcal{X})$, and $T^{(1)} \overline{\operatorname{SVect}(\mathcal{X})}$ the Lie algebra of $T^{(1)} \overline{\operatorname{SDiff}(\mathcal{X})}=\overline{\operatorname{SDiff}(\mathcal{X})} \ltimes \overline{\operatorname{SVect}(\mathcal{X})}$. The extra derivation $I d_{\text {SVect }(\mathcal{X})} \otimes \mathbb{R}(\delta)$ is readily integrated to a 1-parameter group of automorphisms of $T^{(1)} \overline{\operatorname{SDiff}(\mathcal{X})}: F_{s}(\phi+t \xi)=\phi+t e^{s} \xi$. 
And finally, central extensions of $T^{(1)} \operatorname{SDiff}(\mathcal{X})$ and $T^{(1)} \overline{\operatorname{SDiff(\mathcal {X})}}$ are readily obtained from tangent groups in (7.5) and (7.6).

\section{Dynamical interpretation and applications to Magnetohy- drodynamics(MHD)}

It is well known since the pioneering work of V.I.Arnold in the 60's of the last century, that the Lie group of volume preserving diffeomorphisms $\overline{\operatorname{Siff(\mathcal {X})}}$ is the configuration space for incompressible fluid dynamics, volume form $\omega$ being fixed; the vector field of velocities lives in its Lie algebra $\mathfrak{g}=S V$ ect $(\mathcal{X})$, and the Euler equation gives:

$$
\dot{\xi}+\nabla_{\xi} \xi+\nabla p=f
$$

Here $\xi \in \mathfrak{g}$ is the field of velocities in Euler description, $p$ the field of pressure in the fluid, and $f$ the field of external forces, representing all possible kind of external coupling, for example electromagnetic or possibly non abelian gauge interactions. Following the approach of[1], we shall recall how this equation can be made Hamiltonian on the regular dual $S V$ ect $(\mathcal{X})_{\text {reg }}^{*}=\Omega^{1}(\mathcal{X}) / Z^{1}(\mathcal{X})$. Let's choose a metric on $\mathfrak{g}$ independently of the volume form, denoting simply by . the scalar product ; one then constructs the inertia operator $I: \mathfrak{g} \rightarrow \mathfrak{g}^{*}$ naturally defined as $\langle I(\xi), \eta\rangle=\xi . \eta$, the brackets $<,>$ indicate as usual coupling between a vector space and its dual.

This isomorphism depends on the physics of the problem, we can call it dynamical isomorphism in contrast with the kinematical isomorphism $\mathcal{J}^{\sharp}$ previously defined, cf. [1] for details, or[22] for analogous point of view in a slightly different problem. One then defines the energy on $\mathfrak{g}$ as $H(\xi)=\frac{1}{2}\|\xi\|^{2}=\frac{1}{2}\left\langle I(\xi), \xi>\right.$, and dually on $\mathfrak{g}^{*}$ as $H([u])=\frac{1}{2}<[u], I^{-1}[u]>$. Then we can rewrite the Euler equation above as Hamiltonian on the Poisson manifold $\mathfrak{g}^{*}$ (without external forces):

$$
[\dot{u}]+L_{I^{-1}[u]}[u]=0,
$$

in terms of coadjoint action, we obtain the equivalent form, also obviously hamiltonian:

$$
[\dot{u}]+a d_{I^{-1}[u]}^{*}[u]=0
$$

The pressure is then included in the class $[u]$ of a 1 -form $u$ modulo exact ones(cf.[1] p. $38)$.

We shall consider now Magnetohydrodynamics (MHD for short) in which the fluid carries electric charges, submitted to Lorentz forces, and creates its own field through the moving charges. In fact we consider the particular case, when electric field is 
negligible, and magnetic field is frozen in fluid; see [5] for details about MHD. So for matter field $\xi$ and magnetic field $B$, both in $S V$ ect $(\mathcal{X})$ admit evolution equations as follows:

$$
\begin{gathered}
\dot{\xi}+\nabla_{\xi} \xi+\nabla p-\operatorname{curl}(B) \wedge B=0 \\
\dot{B}+[\xi, B]=0
\end{gathered}
$$

We can see now that the configuration space for MHD is the semi-direct product $\operatorname{SDiff}(\mathcal{X}) \ltimes \Omega^{1}(\mathcal{X}) / Z^{1}(\mathcal{X})$, and corresponding Lie algebra is magnetic extension $T^{*} S V$ ect $(\mathcal{X})$. In [1] p.54, the author gives hamiltonian interpretation of MHD equations, on the dual of this Lie algebra, the hamiltonian being

$$
\mathcal{H}([u], B)=\frac{1}{2}<[u], I^{-1}[u]>+\frac{1}{2}<I(B), B>
$$

Set $I(B)=[b]$, we obtain the following formulation:

$$
\begin{gathered}
{[\dot{u}]+L_{I^{-1}[u]}[u]-L_{B}[b]=0} \\
\dot{B}+\left[I^{-1}[u], B\right]=0
\end{gathered}
$$

Equivalently, one can check:

$$
([\dot{u}], \dot{B})+a d_{\left(I^{-1}[u], I(B)\right)}^{*}([u], B)=0 .
$$

From the expression of hamiltonian $\mathcal{H}$ above, one deduces easily:

$$
\frac{\delta \mathcal{H}}{\delta[u]}=I^{-1}[u], \frac{\delta \mathcal{H}}{\delta B}=I(B) .
$$

So one can reformulate MHD equation :

$$
([\dot{u}], \dot{B})+a d_{\left(\frac{\delta \mathcal{H}}{\delta(u)}, \frac{\delta \mathcal{H}}{\delta B}\right)}^{*}([u], B)=0
$$

or in terms of Poisson bracket :

$$
([\dot{u}], \dot{B})+\{\mathcal{H},([u], B)\}=0
$$

This is a hamiltonian equation on $\operatorname{SVect}(\mathcal{X}) \times \Omega^{1}(\mathcal{X}) / Z^{1}(\mathcal{X})$ seen as regular dual of magnetic extension $T^{*} S V$ ect $(\mathcal{X})$, and with hamiltonian $\mathcal{H}$ defined above.

We shall now give our main result, showing how those MHD equations are deformed after double extension of $T^{*} S V e c t(\mathcal{X})$, but without modification of the Hamiltonian (the dynamics). We shall use formulas (8.4) and (8.5) above, transposed to $T^{*} S V e c t(\mathcal{X})$ through kinematical isomorphism:

$$
([\dot{u}], \dot{B})+\tilde{a} d_{\left(\left(I^{-1}[u], I(B)\right), \lambda \delta+(U, W)\right)}^{*}(([u], B),(Z, z))=0 .
$$


We obtain :

\section{Theorem:}

The following system is hamiltonian on the dual of magnetic extension $T^{*} S V e c t(\mathcal{X})$ with hamiltonian $\mathcal{H}$ defined as in formula (8.3) above

$$
\begin{gathered}
{[\dot{u}]+L_{\xi}[u]-L_{B}[b]+i_{\xi}[H]+i_{B}[h]+L_{U}[u]=0} \\
\dot{B}+[\xi, B]+\xi \wedge h^{\sharp}+\lambda B+[U, B]+[W, \xi]=0
\end{gathered}
$$

(Remark: we have made $Z=1$ and $z=1$ by entering the central charges as coefficients of the 2 -forms $H$ and $h$; recall $I^{-1}[u]=\xi$ and $\left.i_{h^{\sharp}}(\omega)=h\right)$. The 2 -form $d u$ is interpreted as the vorticity of the fluid, cf. $[1,15]$.

Those equations can be rewritten in a more concise form

$$
\begin{gathered}
{[\dot{u}]+L_{\xi+U}[u]-L_{B}[b]+i_{B}[h]+i_{\xi}[H]=0} \\
\dot{B}+[\xi+U, B-W]+\xi \wedge h^{\sharp}+\lambda B=0 .
\end{gathered}
$$

Recall that $[U]$ and $[W]$ are classes in the quotient $\overline{\operatorname{SVect}}(\mathcal{X})$ modulo $\operatorname{SVect}(\mathcal{X})$,so $[U, W]=0$.

One can also give a purely contravariant formulation, closer to the physical signification of MHD equations:

$$
\begin{gathered}
\dot{\xi}+\nabla_{\xi} \xi+\nabla p-\operatorname{curl}(B) \wedge B+\xi \wedge H^{\sharp}+B \wedge h^{\sharp}+[U, \xi]=0 \\
\dot{B}+[\xi, B]+\xi \wedge h^{\sharp}+\lambda B+[U, B]+[W, \xi]=0
\end{gathered}
$$

\section{Particular cases:}

In the case when topology is trivial, i.e. when $H_{d R}^{1}(\mathcal{X})=H_{d R}^{2}(\mathcal{X})=0$, the above system reduces to

$$
\begin{gathered}
\dot{\xi}+\nabla_{\xi} \xi+\nabla p-\operatorname{curl}(B) \wedge B=0 \\
\dot{B}+[\xi, B]+\lambda B=0
\end{gathered}
$$

The term in $\lambda B$ changes the solution from $B$ into $\exp (-\lambda t) B$, so one gets a kind of dampening term when $\lambda>0$.

Suppose now that $H_{d R}^{1}(\mathcal{X})$ and $H_{d R}^{2}(\mathcal{X})$ are non trivial, the term $i_{\xi}[H]$ or $\xi \wedge h^{\sharp}$ can be interpreted as the introduction of an external magnetic field $H$ constant in time; in purely hydrodynamic case (i.e. if $B=0$ ) this equation is considered in [1] pp 325-326 as infinite conductivity equation for electron gas. In order to give some 
interpretation for external derivation terms, we shall suppose $\mathcal{X}=\mathbb{T}^{3}$; the quotient $\overline{S V e c t}(\mathcal{X}) / S V e c t(\mathcal{X})$ is then 3 -dimensional generated by classes of vector fields $\partial / \partial x$, $\partial / \partial y$ and $\partial / \partial z$. Let's choose for example $U=\partial / \partial x$ and $W=\partial / \partial y$, the equations in contravariant form can then be rewritten as:

$$
\begin{gathered}
\dot{\xi}+\nabla_{\xi} \xi+\nabla p-\operatorname{curl}(B) \wedge B+\xi \wedge H^{\sharp}+B \wedge h^{\sharp}+\partial \xi / \partial x=0 \\
\dot{B}+[\xi, B]+\xi \wedge h^{\sharp}+\lambda B+\partial B / \partial x+\partial \xi / \partial y=0
\end{gathered}
$$

or:

$$
\begin{gathered}
(\partial / \partial t+\partial / \partial x) \xi+\nabla_{\xi} \xi+\nabla p-\operatorname{curl}(B) \wedge B+\xi \wedge H^{\sharp}+B \wedge h^{\sharp}=0 \\
(\partial / \partial t+\partial / \partial x) B+[\xi, B]+\xi \wedge h^{\sharp}+\lambda B+\partial \xi / \partial y=0
\end{gathered}
$$

So the terms corresponding to external derivations induce some change in time as if $t \rightarrow t+x$; this could perhaps have a relativistic interpretation.

Remark: One can go one step further using group theory applied to symplectic geometry. All our Poisson structures are Lie-Poisson on the dual of Lie algebras; it is well known that linear Poisson structure on $\mathfrak{g}^{*}$ can be obtained from canonical Liouville symplectic structure on cotangent group $T^{*} G$ by symplectic reduction through natural action of $G$ by translations, left or right as $\underset{\hat{\tilde{n}}}{\operatorname{explained}}$ in [21] part 2. So to our group integrating the double extension $\mathcal{G}=T^{(1)} \hat{\operatorname{SDiff}} f(\mathcal{X})$ is associated its cotangent $T^{*} \mathcal{G}$, and our dynamical system can be lifted to $T^{*} \mathcal{G}$ as an hamiltonian system for the canonical symplectic structure. Moreover the corresponding hamiltonian $\mathcal{H}$ naturally defines a metric on the group, for which our equation is Euler, just like for MHD, as proved in [1].

\section{A supersymmetric analogue}

For basic definitions and constructions on Lie superalgebras, see[26], and for some notions in supergeometry, see the beautiful book [7]. We shall describe the super version of Kac-Moody algebras, first constructed by V.Kac.

To any Lie algebra $\mathfrak{g}$ one can associate a Lie superalgebra $\overline{\mathfrak{g}}$ defined as follows: Consider first $\mathfrak{g}_{\theta}=\mathfrak{g} \otimes \Lambda(\theta)$, where $\theta$ is an odd variable, and the bracket is then naturally defined as

$$
[X+\theta x, Y+\theta y]=[X, Y]+\theta[x, Y]+\theta[X, y]
$$

then, as a $\mathbb{K}$-vector space, and $\overline{\mathfrak{g}}=\mathfrak{g}_{\theta} \oplus \mathbb{K} \delta$, the bracket with $\delta$ being

$$
[X, \delta]=0,[\delta, \theta x]=x .
$$


In other words $\overline{\mathfrak{g}}_{0}=\mathfrak{g}, \overline{\mathfrak{g}}_{1}=\mathfrak{g}_{\theta} \oplus \mathbb{K} \delta$, and in supergeometric terms, $\delta$ acts as $\partial / \partial \theta$. If now $\mathfrak{g}$ is semisimple with Killing form $\kappa$, one can mimic the construction of Kac-Moody central cocycle in this graded context, the integral being the Berezin integral Ber[7]. One then obtains:

$$
c(u, v)=\int_{0 \mid 1} \kappa(u, \delta(v)) B e r,
$$

more explicitly if $u=X+\theta x, v=Y+\theta y$, then:

$$
c(u, v)=\kappa(x, y) .
$$

This 2-cocycle is of odd-odd type, hence its symmetry, surprising at first glance. So one finally obtains a double extension, just as in the even case:

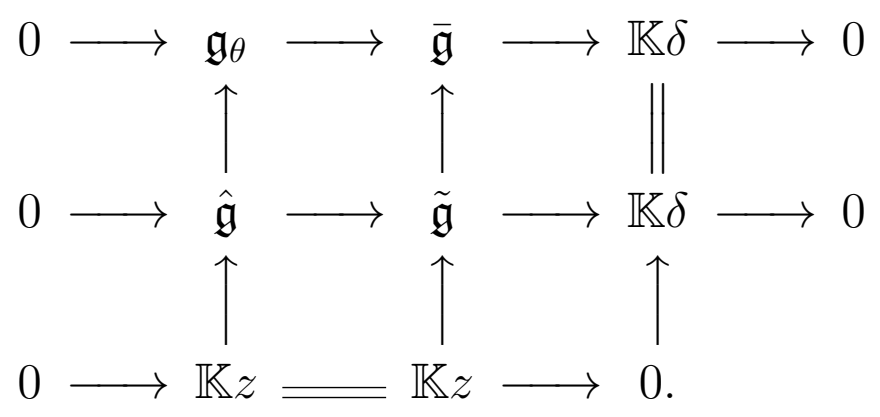

Moreover the Killing form extends naturally to $\hat{\mathfrak{g}}$, as

$$
\hat{\kappa}(u, v)=\int_{0 \mid 1} \kappa(u, v) B e r
$$

which gives $\hat{\kappa}(u, v)=\kappa(X, y)+\kappa(x, Y)$, if $u=X+\theta x, v=Y+\theta y$ as above. One sets $\hat{\kappa}(\delta, z)=1$, and other terms equal to zero. This allow identification between $\hat{\mathfrak{g}}$ and its dual. The odd and even part are easily identified: $\hat{\mathfrak{g}}_{0}=\mathfrak{g} \oplus \mathbb{K} z$, and $\hat{\mathfrak{g}}_{1}=$ $\mathfrak{g}_{\theta} \oplus \mathbb{K} \delta$, so dimension of odd and even part are equal, and one of the main axioms of supersymmetry is thus satisfied(cf.[30]).

One can try generalization to higher dimensions; let $\mathcal{A}_{N}$ be the exterior algebra on $N$ generators and set $\mathfrak{g}_{N}=\mathfrak{g} \otimes \mathcal{A}_{N}$. One has

$$
H^{1}\left(\mathfrak{g}_{N}, \mathfrak{g}_{N}\right)=\operatorname{Der}\left(\mathcal{A}_{N}\right)=\operatorname{Vect}(0 \mid N) .
$$

The first equality being a particular case of formula (3.1), the second one comes from the geometrical interpretation of $\mathcal{A}_{N}$ as the algebra of functions on superspace of dimension $0 \mid N$, its superderivations being given by the Lie superalgebra of vector fields on the same superspace. We must mention here that the Lie superalgebra $V e c t(0 \mid N)$ plays a key role in algebraic deformation theory under the name of Richardson-Nijenhuis algebra. For central extensions, one can use the formula(3.2) in its graded version:

$$
H_{2}\left(\mathfrak{g}_{N}\right)=H C_{1}\left(\mathcal{A}_{N}\right)=\Omega^{1}\left(\mathcal{A}_{N}\right) / d \mathcal{A}_{N}
$$


So one can construct central extension and extension by derivations for $\mathfrak{g}_{N}$

$$
\begin{aligned}
0 \longrightarrow & \Omega^{1}\left(\mathcal{A}_{N}\right) / d \mathcal{A}_{N} \stackrel{i}{\longrightarrow} \hat{\mathfrak{g}}_{N} \stackrel{\pi}{\longrightarrow} \mathfrak{g}_{N} \longrightarrow 0 \\
0 \longrightarrow & \longrightarrow \mathfrak{g}_{N} \stackrel{i}{\longrightarrow} \overline{\mathfrak{g}}_{N} \stackrel{\pi}{\longrightarrow} \operatorname{Vect}(0 \mid N) \longrightarrow 0
\end{aligned}
$$

In[6] the central extensions of $\mathfrak{g}_{N}$ are determined through calculations by hand. We shall interpret them using the dual version of (3.2)

$$
H^{2}\left(\mathfrak{g}_{N}\right)=H C^{1}\left(\mathcal{A}_{N}\right),
$$

one can compute cyclic cohomology using Connes sequence:

$$
H C^{1}\left(\mathcal{A}_{N}\right)=\operatorname{ker} \partial: H H^{1}\left(\mathcal{A}_{N}\right) \rightarrow H H^{0}\left(\mathcal{A}_{N}\right) .
$$

Here $H H^{*}$ stands for Hochschild cohomology, which gives $H H^{1}\left(\mathcal{A}_{N}\right)=\operatorname{Vect}(0 \mid N)$ and $H H^{0}\left(\mathcal{A}_{N}\right)=\mathcal{A}_{N}$. Computations in [6] enables to identify operator $\partial$ with superdivergence of supervector fields; so $H C^{1}\left(\mathcal{A}_{N}\right)$ turns out to be isomorphic to superalgebra $\operatorname{SVect}(0 \mid N)$ of vector fields in dimension $0 \mid N$ with vanishing superdivergence. One remarks the duality between cyclic homology and cohomology as follows:

$$
\begin{aligned}
& \mathcal{A}_{N}=H H_{0}\left(\mathcal{A}_{N}\right) \stackrel{d}{\longrightarrow} \Omega^{1}\left(\mathcal{A}_{N}\right)=H H_{1}\left(\mathcal{A}_{N}\right) \stackrel{\pi}{\longrightarrow} \Omega^{1}\left(\mathcal{A}_{N}\right) / d \mathcal{A}_{N}=H C^{1}\left(\mathcal{A}_{N}\right) \underset{(9.3)}{\longrightarrow} 0 \\
& 0 \longrightarrow \operatorname{SVect}(0 \mid N)=H C^{1}\left(\mathcal{A}_{N}\right) \stackrel{i}{\longrightarrow} \operatorname{Vect}(0 \mid N)=H H^{1}\left(\mathcal{A}_{N}\right) \stackrel{\partial}{\longrightarrow} \mathcal{A}_{N}=H H^{0}\left(\mathcal{A}_{N}\right)
\end{aligned}
$$

computations being direct generalizations of the even case (see [20]). So one obtains a seemingly nice duality between extensions (9.2) and (9.3) above, when the latter is restricted to $S V \operatorname{ect}(0 \mid N)$. But unfortunately, the compatibility condition in part 2 above is satisfied only when derivations commute with each other, so one can expect double extension generalizing the $N=1$ case only with abelian subalgebras of $S V \operatorname{ect}(0 \mid N)$ and their corresponding duals in $\Omega^{1}\left(\mathcal{A}_{N}\right) / d \mathcal{A}_{N}$. The principle of SUSY which imposes the same number of odd and even dimensions is still respected.

For kinematical and dynamical interpretation, one would have first to investigate the supergeometry of coadjoint orbits of those algebras; curiously, very few is known on that topic, save for the pionniering works of G.Tuynman[28][27].

\section{Final remarks}

In this section we shall set $S V e c t(\mathcal{X})=\mathfrak{g}$ for short; in [18] we studied the deformations of this Lie algebra, but it turns out to be rigid. The only non trivial case is $n=3$ 
where $\operatorname{dim} H^{2}(\mathfrak{g}, \mathfrak{g})=1$, generated by a cohomology class $\Phi$ constructed explicitly below. Unfortunately (or fortunately?) the infinitesimal deformation defined by $\Phi$ doesn't admit any prolongation, hence the rigidity. Nevertheless this infinitesimal deformation defines an actual deformation on $T \mathfrak{g}$ as follows :

$$
[X+t x, Y+t y]=[X, Y]+t[x, Y]+t[X, y]+t \lambda \Phi(X, Y)
$$

the scalar parameter $\lambda$ being arbitrary. Let's recall the construction of $\Phi$ from [18], one has an exact sequence:

$$
0 \longrightarrow \mathfrak{g} \stackrel{i}{\longrightarrow} \Omega^{2}(\mathcal{X}) \stackrel{d}{\longrightarrow} \Omega^{3}(\mathcal{X}) \longrightarrow 0
$$

As above, one has $i(X)=i_{X} \omega, \omega$ being the volume form and $d$ the exterior differential, the exactness of the above sequence being understood in the sense of sheaf theory. We can give now some hints about the construction of $\Phi$; there exist natural cocycles on Lie algebras of vector fields generalizing divergence in higher degrees, they were constructed by I.M Gelfand[8] as early as 1970. We shall need here

$$
\Psi: \operatorname{Vect}(\mathcal{X}) \times \operatorname{Vect}(\mathcal{X}) \rightarrow \Omega^{2}(\mathcal{X}) .
$$

We can give a local formula which will make apparent the fact that we do have generalized the divergence:

$$
\Psi(X, Y) \mid=\partial_{a i} X^{b} \partial_{b j} Y^{a} d x_{i} \wedge d x_{j} .
$$

(Remark: we have used Einstein convention about indices; this formula will sound familiar to all those interested in deformation quantization, it is a version of Vey cocycle, the key element for construction of star-products). We can now construct $\Phi$, by restricting $\Psi$ to $\mathfrak{g}$, and then contracting with the volume tensor dual to $\omega$; we give here only the basic ideas, the full proof being much more technical(cf.[18]). We can also transfer this deformation on magnetic extension $T^{*} \mathfrak{g}$, using kinematic isomorphism $\mathcal{J}^{\sharp}$; it was done by Billig in[4] who obtained the following form for the cocycle

$$
\tau: \mathfrak{g} \times \mathfrak{g} \rightarrow \Omega^{1}(\mathcal{X}) / Z^{1}(\mathcal{X}),
$$

defined locally as

$$
\tau(X, Y) \mid=\left[\partial_{a} X^{b} d\left(\partial_{b} Y^{a}\right)\right] .
$$

He makes the link with his earlier works about abelian ( i-e, non central) extensions[3] called toroidal Lie algebras, and also stresses the analogy with Virasoro cocycle, which is also of order of differentiability 3 .

On the dual of the deformed Lie algebra $T^{*} \mathfrak{g}_{\tau}$, the equations of Magnetohydrodynamics are naturally deformed as a new hamiltonian system:

$$
([\dot{u}], \dot{B})+a d_{(\tau)\left(I^{-1}[u], I(B)\right)}^{*}([u], B)=0 .
$$


In contravariant terms, the deformed equations reads:

$$
\begin{gathered}
\dot{\xi}+\nabla_{\xi} \xi+\nabla p-\operatorname{curl}(B) \wedge B+\operatorname{Div} \mathcal{T}=0 \\
\dot{B}+[\xi, B]=0,
\end{gathered}
$$

where $\mathcal{T}$ is physically interpreted as asymmetric stress tensor, following $\mathcal{T}^{i j}=\frac{\partial B^{j}}{\partial x_{a}} \frac{\partial \xi^{a}}{\partial x_{i}}$ and appears in the equations through the deformed cocycle $\tau$, cf.[3] for details.

\section{Appendix}

We shall prove that $H C_{1}(\mathcal{A})=0$ for $\mathcal{A}=\mathbb{K}[t] /\left(t^{2}\right)$ the local ring of dual numbers. Following [12] p.172 sqq., we can determine the Kähler 1-forms explicitly ; let $I=$ ker $\mu: \mathcal{A} \otimes \mathcal{A} \rightarrow \mathcal{A}$, it is 2-dimensional with generators $t \otimes t$ and $1 \otimes t-t \otimes 1$; then $I^{2}$ is generated by $t \otimes t$ and $\Omega^{1}(\mathcal{A})=I / I^{2}$ is one dimensional with $d: \mathcal{A} \rightarrow \Omega^{1}(\mathcal{A})$ an isomorphism. So, $H C_{1}(\mathcal{A})=\Omega^{1}(\mathcal{A}) / d \mathcal{A}=0$. This proof can in fact be generalized to the case of all artinian rings of truncated polynomials $\mathcal{A}_{k}=\mathbb{K}[t] /\left(t^{k}\right)$, see[20], E 4.1.8.

\section{Acknowledgments}

The author thanks those whose conversations were so useful during elaboration of this work, especially Boris Khesin and Tudor Ratiu ; and also Cornelia Vizman and Gijs Tuynman for useful indications. He is grateful to Alice Fialowski and Anne Pichereau for their invitations to lecture on the subject at conferences in Budapest and St-Etienne.

\section{References}

[1] Vladimir I. Arnold and Boris A. Khesin. Topological methods in hydrodynamics, volume 125 of Applied Mathematical Sciences. Springer-Verlag, New York, 1998.

[2] Augustin Banyaga. The structure of classical diffeomorphism groups, volume 400 of Mathematics and its Applications. Kluwer Academic Publishers Group, Dordrecht, 1997.

[3] Yuly Billig. Abelian extensions of the group of diffeomorphisms of a torus. Lett. Math. Phys., 64(2):155-169, 2003.

[4] Yuly Billig. Magnetic hydrodynamics with asymmetric stress tensor. J. Math. Phys., 46(4):043101, 13, 2005. 
[5] Dieter Biskamp. Nonlinear magnetohydrodynamics, volume 1 of Cambridge Monographs on Plasma Physics. Cambridge University Press, Cambridge, 1993.

[6] R. Coquereaux, L. Frappat, E. Ragoucy, and P. Sorba. Extended superKac-Moody algebras and their super-derivation algebras. Comm. Math. Phys., 133(1):1-35, 1990.

[7] Pierre Deligne, Pavel Etingof, Daniel S. Freed, Lisa C. Jeffrey, David Kazhdan, John W. Morgan, David R. Morrison, and Edward Witten, editors. Quantum fields and strings: a course for mathematicians. Vol. 1, 2. American Mathematical Society, Providence, RI, 1999. Material from the Special Year on Quantum Field Theory held at the Institute for Advanced Study, Princeton, NJ, 1996-1997.

[8] I. M. Gel'fand. The cohomology of infinite dimensional Lie algebras: some questions of integral geometry. In Actes du Congrès International des Mathématiciens (Nice, 1970), Tome 1, pages 95-111. Gauthier-Villars, Paris, 1971.

[9] Aziz Haddi. Détermination des extensions centrales des algèbres de Kac-Moody. C. R. Acad. Sci. Paris Sér. I Math., 306(16):691-694, 1988.

[10] Aziz Haddi. Homologie des algèbres de Lie étendues à une algèbre commutative. Comm. Algebra, 20(4):1145-1166, 1992.

[11] Stefan Haller and Cornelia Vizman. Non-linear Grassmannians as coadjoint orbits. Math. Ann., 329(4):771-785, 2004.

[12] Robin Hartshorne. Algebraic geometry. Springer-Verlag, New York, 1977. Graduate Texts in Mathematics, No. 52.

[13] P. J. Hilton and U. Stammbach. A course in homological algebra, volume 4 of Graduate Texts in Mathematics. Springer-Verlag, New York, second edition, 1997.

[14] Victor G. Kac. Infinite-dimensional Lie algebras, volume 44 of Progress in Mathematics. Birkhäuser Boston Inc., Boston, MA, 1983. An introduction.

[15] Boris Khesin. Symplectic structures and dynamics on vortex membranes. arXiv:1201.5914v1[math.SG] 27 Jan 2012.

[16] Boris Khesin and Robert Wendt. The geometry of infinite-dimensional groups, volume 51 of Ergebnisse der Mathematik und ihrer Grenzgebiete. 3. Folge. A Series of Modern Surveys in Mathematics. Springer-Verlag, Berlin, 2009.

[17] A. A. Kirillov. Lectures on the orbit method, volume 64 of Graduate Studies in Mathematics. American Mathematical Society, Providence, RI, 2004. 
[18] Pierre B. A. Lecomte and Claude Roger. Rigidité de l'algèbre de Lie des champs de vecteurs unimodulaires. J. Differential Geom., 44(3):529-549, 1996.

[19] André Lichnerowicz. Algèbre de Lie des automorphismes infinitésimaux d'une structure unimodulaire. Ann. Inst. Fourier (Grenoble), 24(3):xiv, 219-266, 1974.

[20] Jean-Louis Loday. Cyclic homology, volume 301 of Grundlehren der Mathematischen Wissenschaften. Springer-Verlag, Berlin, second edition, 1998.

[21] Jerrold E. Marsden and Tudor S. Ratiu. Introduction to mechanics and symmetry, volume 17 of Texts in Applied Mathematics. Springer-Verlag, New York, second edition, 1999. A basic exposition of classical mechanical systems.

[22] V. Ovsienko and C. Roger. Looped cotangent Virasoro algebra and non-linear integrable systems in dimension 2+1. Comm. Math. Phys., 273(2):357-378, 2007.

[23] Claude Roger. Extensions centrales d'algèbres et de groupes de Lie de dimension infinie, algèbre de Virasoro et généralisations. Rep. Math. Phys., 35(2-3):225-266, 1995. Mathematics as language and art (Białowieża, 1993).

[24] Claude Roger. Unimodular vector fields and deformation quantization. In Deformation quantization (Strasbourg, 2001), volume 1 of IRMA Lect. Math. Theor. Phys., pages 135-148. de Gruyter, Berlin, 2002.

[25] Claude Roger. The group of volume preserving diffeomorphisms and the Lie algebra of unimodular vector fields: survey of some classical and not-so-classical results. In Twenty years of Bialowieza: a mathematical anthology, volume 8 of World Sci. Monogr. Ser. Math., pages 79-98. World Sci. Publ., Hackensack, NJ, 2005.

[26] Manfred Scheunert. The theory of Lie superalgebras, volume 716 of Lecture Notes in Mathematics. Springer, Berlin, 1979. An introduction.

[27] G. M. Tuynman. Geometric quantization of superorbits: a case study. arXiv:0901.1811, (12), 2009.

[28] G. M. Tuynman. Super symplectic geometry and prequantization. J. Geom. Phys., 60(12):1919-1939, 2010.

[29] Cornelia Vizman. Lichnerowicz cocycles and central Lie group extensions. An. Univ. Vest Timis. Ser. Mat.-Inform., 48(1-2):285-297, 2010.

[30] P. West. Introduction to supersymmetry and supergravity. World Scientific Publishing Co., Singapore, 1986. 
[31] Paul Zusmanovich. The second homology group of current Lie algebras. Astérisque, (226):11, 435-452, 1994. K-theory (Strasbourg, 1992). 